\title{
Comparison of acidic and enzymatic pectin extraction from passion fruit peels and its gel properties
}

\begin{abstract}
The influences of extractor concentration, extraction temperature and time on the yield of pectin and degree of esterification (DE) were investigated by the acidic and enzymatic extraction methods. Citric acid and Celluclast were selected as pectin extractors for environmentally friendly reasons. The peels of yellow passion fruit using the acidic and enzymatic extraction methods gave pectin yield of 7.16 and $7.12 \%$, and DE of 71.02 and $85.45 \%$ in the optimized condition of extraction time of $102 \mathrm{~min}$ with citric acid concentration of $0.19 \%(\mathrm{w} / \mathrm{w})$ at $75 \mathrm{C}$ and Celluclast concentration of $1.67 \%(\mathrm{w} / \mathrm{w})$ at $61.11 \mathrm{C}$, respectively. The enzymatic extraction method has greater capability of producing high methoxyl pectin. The morphological features of fruit peel powder and the extracted pectin examined by scanning electron microscopy suggested that the nanostructure of wet passion fruit pectin was dependable on the type of extraction process. The formed pectin gel also has pseudoplastic liquid behavior and its viscosity was greatly affected by sugar. Pectin has been intensively used as natural gelling agent and stabilizer to alter rheological properties in food ingredients by most food processing industries to achieve desired textural quality. Pectin could be recovery from fruit wastes. The conversion of passion fruit peel into pectin offers great scope for utilization. Citric acid and enzymatic extraction methods are effectively used for pectin extraction which may be of interest by pectin industry and consumer with these eco-friendly processing technology with no using harmful chemicals. Furthermore, scientific work of this study such as the optimized condition, morphological features of extracted pectin and pectin gel formation contributes valuable information on pectin, which could be beneficial for pectin industry improving the process quality of pectin as well as process profitability.
\end{abstract}

Keyword: Acidic; Enzymatic; The yellow passion fruit; Dietry 